\title{
The ideal duplication
}

\section{Danny Troia ${ }^{1}$}

Received: 14 November 2020 / Accepted: 1 June 2021 / Published online: 21 June 2021

(c) The Author(s) 2021

\begin{abstract}
In this paper we present and study the ideal duplication, a new construction within the class of the relative ideals of a numerical semigroup $S$, that, under specific assumptions, produces a relative ideal of the numerical duplication $S \bowtie^{b} E$. We prove that every relative ideal of the numerical duplication can be uniquely written as the ideal duplication of two relative ideals of $S$; this allows us to better understand how the basic operations of the class of the relative ideals of $S \bowtie^{b} E$ work. In particular, we characterize the ideals $E$ such that $S \bowtie^{b} E$ is nearly Gorenstein.
\end{abstract}

Keywords Nearly Gorenstein semigroups · Numerical duplication · Relative ideal · Canonical ideal

\section{Introduction}

The numerical duplication is a construction introduced in [7] that, starting with a numerical semigroup $S$ and a semigroup ideal $E \subset S$, produces a new numerical semigroup, denoted by $S \bowtie^{b} E$ (where $b$ is any odd integer belonging to $S$ ). The origin of this construction is connected to ring theory; more precisely, in [2] it is studied a family of quadratic quotients of the Rees algebra of a ring $R$ with respect to an ideal $I$, with the aim of giving a unified approach for Nagata's idealization (see e.g. Nagata's book [13, p. 2] and [8]) and amalgamated duplication (see e.g. [5,6]); when the original ring $R$ is a numerical semigroup ring or an algebroid branch, so it has a value semigroup $S=v(R)$, particular members of the family are again numerical semigroup rings (or, respectively, algebroid branches) whose value semigroup is a numerical duplication of $S$.

Moreover, if $R$ is local, some relevant properties, such as Gorensteinness, almost Gorensteinness and Cohen-Macaulay type, coincide for any member of the family cited

\section{Communicated by Jorge Almeida.}

Danny Troia

danny.troia@phd.unict.it

1 Dipartimento di Matematica e Informatica, Viale A. Doria 6, 95125 Catania, Italy 
above, depending only on $R$ and $I$ (see [3]); in [7] it is shown that the same happens for numerical duplication. In particular, it is given a characterization of those ideals that produce a symmetric or an almost symmetric semigroup; this characterization, as expected, does not depend on the integer $b$ used to define the numerical duplication.

In this paper we want to provide a systematical study of relative ideals of a numerical duplication; to this aim we introduce a similar construction for relative ideals, that we call ideal duplication: given a numerical semigroup $S$, an odd number $b \in S$ and an ideal $E$, the ideal duplication starting from two relative ideals $E_{1}, E_{2}$ of $S$, under specific assumptions, produces a relative ideal $E_{1} \bowtie^{b} E_{2}$ of the numerical duplication $S \bowtie^{b} E$. The first main result of this paper (see Theorem 3.4) is that every relative ideal of the numerical duplication can be written, in a unique way, as the ideal duplication of two relative ideals of the semigroup.

The knowledge of the relative ideals of a numerical duplication allows to better understand its properties; for example, we will be able to describe the trace of the numerical duplication (see Theorem 4.2) and its pseudo-Frobenius numbers (see Theorem 4.12). The trace of a numerical semigroup is connected to the nearly Gorenstein property, that is the numerical analogue of a ring property, originally introduced by Herzog, Hibi and Stamate [11], using the trace of the canonical module. Nearly Gorenstein rings generalize Gorenstein rings, and this class is, in general, independent of the class of almost Gorenstein rings. However, in the one-dimensional case, almost Gorenstein rings are nearly Gorenstein. Hence, the class of nearly Gorenstein semigroups generalizes the class of almost symmetric semigroups; so, as it was done for the almost symmetric case, it is natural to look for a characterization of those ideals $E$, such that $S \ltimes^{b} E$ is nearly Gorenstein. Using Theorem 4.2, we are able to find this characterization (see Corollary 4.3).

The structure of the paper is the following: in Sect. 2 we recall all the basic notions on numerical semigroups, that we will use in the rest of the paper, and we prove some preliminary lemmas about relative ideals.

In Sect. 3, we define the ideal duplication, we show that every relative ideal of the numerical duplication is the ideal duplication of two relative ideals of the semigroup (see Theorem 3.4) and we apply this fact to better understand relative ideals of the numerical duplication and the basic operations between them (e.g. sum, difference, shift, etc.); moreover, we describe, in terms of ideal duplication, the canonical ideal of the numerical duplication (see Proposition 3.5).

In Sect. 4, using the ideal duplication, we are able to describe the trace of the numerical duplication (see Theorem 4.2) and, from this, it follows a characterization of the nearly Gorenstein property (see Corollary 4.3); furthermore, we apply this result to study the nearly Gorensteinness for numerical duplications obtained by some particular classes of ideals, like e.g. integrally closed ideals (see Theorem 4.11). Finally, we give a description of the pseudo-Frobenius numbers of the numerical duplication (see Theorem 4.12) which produces a new characterization for $S \bowtie^{b} E$ to be almost symmetric (see Corollary 4.13). 


\section{Preliminaries}

Most of the definitions and results that we recall in this section can be found in [10] or in [1]; if not, we will give specific references. A numerical semigroup $S$ is an additive submonoid of $\mathbb{N}$, such that $|\mathbb{N} \backslash S|<\infty$. The elements of $\mathbb{N} \backslash S$ are called gaps of $S$ and their cardinality is called the genus of the semigroup, denoted by $g(S)$. The largest gap is called Frobenius number of $S$ and it is denoted by $f(S)$.

It is clear that, if $s \in S$, then $f(S)-s \notin S$. Using this remark, the gaps of $S$ are usually classified as gaps of the first type, i.e. non-negative integers $x \notin S$ of the form $x=f(S)-s$ with $s \in S$, and gaps of the second type, i.e. non-negative integers $y \notin S$, such that $f(S)-y \notin S$. Moreover, if a gap $g$ satisfies $g+s \in S$ for every non-zero $s \in S$, then $g$ is called pseudo-Frobenius. We denote with $\operatorname{PF}(S)$ the set of all pseudo-Frobenius numbers; the type of $S$, denoted by $t(S)$, is the cardinality of $\operatorname{PF}(S)$ and, since $f(S) \in \operatorname{PF}(S), t(S) \geq 1$. A numerical semigroup $S$ is said to be symmetric if there are not gaps of the second type, i.e. $s \in S$ if and only if $f(S)-s \notin S$. It is well known that $\mathrm{S}$ is symmetric if and only if $2 g(S)=f(S)+1$; another equivalent condition for $S$ to be symmetric is $t(S)=1$.

Every numerical semigroup can be written as $\left\{0, a_{1}, \ldots, a_{n}=f(S)+1, \rightarrow\right\}$, with $0<a_{i}<a_{i+1}$; the arrow means that in the set there are all the numbers greater than $f(S)+1$. Moreover, $f(S)+1$ is called the conductor number of $S$.

It is well known that every numerical semigroup is minimally generated by a finite number of elements of $S$, so we will write $S=\left(s_{1}, \ldots, s_{d}\right)$, with $s_{i}<s_{i+1} ; s_{1}$ is called the multiplicity of $S$ and we call $d$ the embedding dimension of $S$. It is easy to prove that $d \leq s_{1}$.

If $S$ is a numerical semigroup, the semigroup ring associated to $S$ is the ring of the form $k[[S]]=k\left[\left[X^{s} \mid s \in S\right]\right] \subseteq k[[X]]$, where $k$ is a field. Many invariants are shared between these two structures (e.g embedding dimension, multiplicity, etc.).

A subset $I$ of $\mathbb{Z}$ is a relative ideal of $S$, if, for every $x \in I$ and $s \in S$, we have $x+s \in I$, and there exists an element $y \in S$ such that $I+y \subseteq S$. If $I \subseteq S$, then we call $I$ an ideal of $S . M(S):=S \backslash\{0\}$ and $C(S):=\{f(S)+1, \rightarrow\}$ are ideals of $S$ and they are respectively the maximal ideal and the conductor ideal of $S$. We can operate with relative ideals obtaining again relative ideals: if $I$ and $J$ are relative ideals of $S$, then $I+J=\{i+j \mid i \in I, j \in J\}$ and $I-J=\{z \in \mathbb{Z} \mid z+j \in I, \forall j \in J\}$ are relative ideals. Moreover, the union and intersection of relative ideals still give relative ideals. We list some properties which will be useful later in the paper.

Lemma 2.1 Let I, J, and $H$ be relative ideals of $S$ and $z \in \mathbb{Z}$. The following statements are true:

1. If $I \subseteq J$, then $I-H \subseteq J-H$ and $H-J \subseteq H-I$;

2. $(I-J)+(J-H) \subseteq I-H$;

3. $(I-J)+H \subseteq(I+H)-J$;

4. $I-(J+H)=(I-J)-H$;

5. $I-(J \cup H)=(I-J) \cap(I-H)$;

6. $I+(J \cup H)=(I+J) \cup(I+H)$;

7. $(I \backslash J)+z=(I+z) \backslash(J+z)$;

8. $(I \cap J)+z=(I+z) \cap(J+z)$. 
Proof All the listed properties are easy to prove, so we include only some of them:

2. Let $x \in I-J$ and $y \in J-H$, then $(x+y)+h=x+(y+h) \in I, \forall h \in H$.

3. Let $x \in I-J$ and $h \in H$, then $(x+h)+j=(x+j)+h \in I+H, \forall j \in J$.

4. Let $x \in I-(J+H)$ and $j \in J$ and $h \in H$, then $(x+h)+j=x+(j+h) \in I$. Conversely, let $x \in(I-J)-H$. Then $x+(j+h)=(x+h)+j \in I$.

It is worth noticing that all the properties listed above still hold if $I, J$, and $H$ are subsets of $\mathbb{Z}$ not necessarily ideals.

If $I$ is a relative ideal of $S$, we define $m(I)=\min I, f(I)=\max (\mathbb{Z} \backslash I)$ (it is well defined since $m(I)+C(S) \subseteq I), C(I)=\{f(I)+1, \rightarrow\}$ the conductor ideal of $I$ and

$$
g(I)=|(\mathbb{Z} \backslash I) \cap\{m(I), m(I)+1, \ldots, f(I)\}|
$$

(note that, if $I \subseteq \mathbb{N}$, then $|\mathbb{N} \backslash I|=g(I)+m(I)$ ). One can always shift a relative ideal $I$ by adding to it an integer $z: z+I=\{z+i \mid i \in I\}$. It is obvious that the relation $I \sim I^{\prime} \Leftrightarrow I^{\prime}=z+I$ for some $z \in \mathbb{Z}$, is an equivalence relation. In every equivalence class there is exactly one representative $\tilde{I}$ such that $f(\tilde{I})=f(S)$. This representative $\tilde{I}$ is obtained by adding $f(S)-f(I)$ to $I$. For all ideals $I$ we have that $C(S) \subseteq \tilde{I} \subseteq \mathbb{N}$.

The set $\{z \in \mathbb{Z} \mid f(S)-z \notin S\}$, denoted by $K(S)$, is a relative ideal of $S$. The following well-known result is usually referred as Jäger's Lemma (see [12, Hilfssatz 5]).

Lemma 2.2 For every relative ideal I of $S$,

$$
K(S)-I=\{x \in \mathbb{Z} \mid f(S)-x \notin I\} .
$$

Proof Let $x \in K(S)-I$ and assume $f(S)-x \in I$. Then $f(S)=(f(S)-x)+x \in$ $K(S)$ yields a contradiction.

Conversely, let $x \in \mathbb{Z}$ such that $f(S)-x \notin I$. Hence, for every $i \in I, f(S)-(x+$ i) $\notin S$, then $x+i \in K(S)$, i.e., $x \in K(S)-I$.

Some consequences of Jäger's Lemma are the following:

Corollary 2.3 The following properties hold for every numerical semigroup:

1. For every relative ideal $I$ of $S, K(S)-(K(S)-I)=I$;

2. $K(S)-K(S)=S$;

3. $K(S)-S=K(S)$;

4. If $I$ and $J$ are relative ideals such that $I \subseteq J$, then $K(S)-J \subseteq K(S)-I$;

5. If $I$ and $J$ are relative ideals such that $I \subseteq J$, then $|J \backslash I|=\mid(K(S)-I) \backslash$ $(K(S)-J) \mid$.

Proof Applying two times Lemma 2.2, we easily prove 1. Properties 2, 3 and 4 are a trivial consequence of Lemma 2.2 and the definition of $K(S)$. Finally, using the map $x \mapsto f(S)-x$ defined in $\mathbb{Z}$ and Lemma 2.2 , we can easily prove 5 . 
Definition 2.4 A relative ideal $I$ of $S$ is said to be canonical if there exists $x \in \mathbb{Z}$ such that $I=x+K(S)$.

The relative ideal $K(S)$ is called the standard canonical ideal of $S$, and it is in fact the representative ideal of the canonical class $[K(S)]$ such that $f(K(S))=f(S)$. It is straightforward to prove that $S \subseteq K(S) \subseteq \mathbb{N}$ and that $S$ is symmetric if and only if $K(S)=S$. It can be proven (see [1, Theorem 3]) that a relative ideal $I$ is canonical if and only if $I-(I-J)=J$, for every relative ideal $J$ of $S$.

The following result will be useful later in the paper, for its easy proof we refer to [1, p. 34].

Proposition 2.5 Let $K$ be the standard canonical ideal of $S$, then, the following is true:

$$
K-M(S)=K \cup\{f(S)\} .
$$

In the paper we will need to use the following facts that we did not find in the literature:

Lemma 2.6 Let I and $J$ be two relative ideals of $S$. Then:

1. $m(I+J)=m(I)+m(J)$;

2. $f(I-J)=f(I)-m(J)$.

Moreover, if $K$ is the standard canonical ideal of $S$, we have:

$$
m(K-I)=f(S)-f(I)
$$

Proof Property 1 is trivial. As for Property 2, notice that $f(I)-m(J) \notin I-J$, but at the same time if $x>0$, then $f(I)-m(J)+x \in I-J$. For the last part, using Lemma 2.2, we have $f(S)-f(I) \in K-I$, also, if $z \in K-I$, then $f(S)-z \leq f(I)$, so $f(S)-f(I) \leq z$.

Proposition 2.7 Let $I$ and $J$ be two relative ideals of $S$ and let $K$ be its standard canonical ideal. The following equality holds:

$$
I-J=(K-J)-(K-I) .
$$

Proof By Property 1 of Corollary 2.3 and by Property 4 of Lemma 2.1, we have:

$$
I-J=(K-(K-I))-J=K-((K-I)+J)=(K-J)-(K-I) .
$$

Corollary 2.8 Let $I$ be a relative ideal of a numerical semigroup $S$ and let $K$ be its standard canonical ideal. The following equality holds:

$$
S-(K-I)=I-K .
$$


Proof Using Proposition 2.7 and both Property 1 and 3 of Corollary 2.3, it follows that

$$
S-(K-I)=((K-(K-I))-(K-S))=I-K .
$$

Corollary 2.9 Let I be a relative ideal of a numerical semigroup $S$ and let $K$ be its standard canonical ideal. Then, $K-\tilde{I}$ is a numerical semigroup if and only if $K-\tilde{I}=I-I$.

Proof If $K-\tilde{I}$ is a numerical semigroup, using Proposition 2.7, we get:

$$
K-\tilde{I}=(K-\tilde{I})-(K-\tilde{I})=\tilde{I}-\tilde{I}=I-I .
$$

The converse statement is trivial.

Notation 2.10 We fix now the notation for the rest of the paper: $S$ is a numerical semigroup, $f$ its Frobenius number, $s_{1}$ its multiplicity, $b \in S$ is an odd number, $E$ is a proper ideal of $S$ (i.e. an ideal such that $E \neq S$ or equivalently $0 \notin E$ ), $e=f(E)-f$, $\tilde{E}=E-e, K$ is the standard canonical ideal of $S$, while $M$ and $C$ are respectively the maximal ideal and the conductor ideal of $S$. For any subset $A \subseteq \mathbb{Z}$, we also define $2 \cdot A=\{2 a \mid a \in A\}$ (note that $2 \cdot S \neq 2 S=S+S$ and $2 \cdot E \neq 2 E=E+E$ ).

\section{Ideal duplication}

In [7, p. 153] is defined the numerical duplication, $S \bowtie^{b} E$, of $S$ with respect to $E$ and $b$ as the following subset of $\mathbb{N}$ :

$$
S \bowtie^{b} E=2 \cdot S \cup(2 \cdot E+b) .
$$

It is straightforward to prove that $S \bowtie^{b} E$ is a numerical semigroup and $f\left(S \bowtie^{b} E\right)=$ $2 f(E)+b$. It is also true that $S \bowtie^{b} E$ is symmetric if and only if $E$ is a canonical ideal of $S$ (see [7, Proposition 3.1.3]).

In this section we define the ideal duplication, an operation between two relative ideals of $S$ which gives, under specific assumptions, a relative ideal of the numerical duplication $S \bowtie^{b} E$. We will prove that every relative ideal of $S \bowtie^{b} E$ can be written, in a unique way, as the ideal duplication of two ideals of $S$. Thanks to this representation, we will be able to better understand how the basic operations among relative ideals of $S \bowtie^{b} E$ work.

Definition 3.1 Let $E_{1}$ and $E_{2}$ be two relative ideals of $S$ satisfying the following:

1. $E_{1}+E \subseteq E_{2}$;

2. $E_{2}+b+E \subseteq E_{1}$.

We define the ideal duplication of $E_{1}$ and $E_{2}$ with respect to $b$ the following set:

$$
E_{1} \bowtie^{b} E_{2}:=2 \cdot E_{1} \cup\left(2 \cdot E_{2}+b\right) .
$$


In the following, when we write $E_{1} \bowtie^{b} E_{2}$, we will always assume that $E_{1}$ and $E_{2}$ (the order is important) satisfy the conditions in Definition 3.1.

Proposition 3.2 The ideal duplication $E_{1} \bowtie^{b} E_{2}$ is a relative ideal of $S \bowtie^{b} E$. Moreover, if $E_{1}$ and $E_{2}$ are proper ideals of $S$ with $E_{2} \subseteq E$, then $E_{1} \bowtie^{b} E_{2}$ is a proper ideal of $S \bowtie^{b} E$.

Proof Let $x \in E_{1} \bowtie^{b} E_{2}$ and $y \in S \bowtie^{b} E$. There are four possibilities:

(i) Let $x=2 e_{1}$, with $e_{1} \in E_{1}$ and $y=2 s$, with $s \in S$. We have:

$$
2 e_{1}+2 s=2\left(e_{1}+s\right) \Rightarrow 2 e_{1}+2 s \in 2 \cdot E_{1} \subseteq E_{1} \bowtie^{b} E_{2} .
$$

(ii) Let $x=2 e_{2}+b$, with $e_{2} \in E_{2}$ and $y=2 s$, with $s \in S$. We have:

$$
2 e_{2}+b+2 s=2\left(e_{2}+s\right)+b \Rightarrow 2 e_{2}+b+2 s \in 2 \cdot E_{2}+b \subseteq E_{1} \bowtie^{b} E_{2} .
$$

(iii) Let $x=2 e_{1}$, with $e_{1} \in E_{1}$, and $y=2 e+b$, with $e \in E$.

$$
2 e_{1}+2 e+b=2\left(e_{1}+e\right)+b \Rightarrow 2 e_{1}+2 e+b \in 2 \cdot E_{2}+b \subseteq E_{1} \bowtie^{b} E_{2} ;
$$

the fact that $e_{1}+e \in E_{2}$ follows from Property 1 of Definition 3.1.

(iv) Let $x=2 e_{2}+b$, with $e_{1} \in E_{1}$, and $y=2 e+b$, with $e \in E$. We have:

$$
2 e_{2}+b+2 e+b=2\left(e_{2}+e+b\right) \Rightarrow 2 e_{2}+b+2 e+b \in 2 \cdot E_{1} \subseteq E_{1} \bowtie^{b} E_{2} ;
$$

the fact that $e_{2}+e+b \in E_{1}$ follows from Property 2 of Definition 3.1.

Since $E_{1}$ and $E_{2}$ are relative ideals, they both have a minimum, hence $2 \cdot E_{1} \cup$ (2 . $E_{2}+b$ ) has a minimum too. Since $S \bowtie^{b} E$ has a conductor, it follows trivially that $\left(E_{1} \bowtie^{b} E_{2}\right)+s \subseteq S \bowtie^{b} E$, for some $s \in S \bowtie^{b} E$.

Example 3.3 Let $S=\{0,3,6, \rightarrow\}, E=\{7,8,10, \rightarrow\}, b=7$. We have

$$
S \bowtie^{7} E=\{0,6,12,14,16,18,20,21,22,23,24,26, \rightarrow\} .
$$

The sets $E_{1}=\{3,6,9, \rightarrow\}$ and $E_{2}=\{-2,1,4, \rightarrow\}$ are relative ideals of $S$.

- $E_{1} \bowtie^{7} E_{2}=\{3,6,9,12,15,17, \rightarrow\}$,

- $E_{2} \bowtie^{7} E_{1}=\{-4,2,8,10,12,13,14,16,18,19,20,22,24, \rightarrow\}$.

$E_{1} \bowtie^{7} E_{2}$ is a relative ideal of $S \rtimes^{7} E$, but $E_{2} \bowtie^{7} E_{1}$ is not (e.g. $-4+21=17 \notin$ $E_{2} \bowtie^{7} E_{1}$ ). We deduce that, in general, in the ideal duplication the order is important.

If $P \subset \mathbb{Z}$ is a subset of even numbers, we call $\frac{P}{2}$ the set of the halves of the numbers in $P$. More precisely

$$
\frac{P}{2}=\{z \in \mathbb{Z} \mid 2 z \in P\}
$$


Theorem 3.4 Let $H$ be a relative ideal of $S \bowtie^{b} E$, then there exist and are unique $E_{1}$ and $E_{2}$ relative ideals of $S$ such that:

$$
H=E_{1} \bowtie^{b} E_{2} .
$$

Moreover, if $H$ is a proper ideal of $S \bowtie^{b} E$, then $E_{1}$ and $E_{2}$ are proper ideals of $S$ with $E_{2} \subseteq E$.

Proof Let $P$ be the set of even numbers of $H$ and let $D$ be the set of odd numbers of $H$. It is trivial to prove:

$$
H=2 \cdot \frac{P}{2} \cup\left(2 \cdot \frac{D-b}{2}+b\right) \text {. }
$$

We want to prove the thesis for $E_{1}=\frac{P}{2}$ and $E_{2}=\frac{D-b}{2}$.

We need to show that $\frac{P}{2}$ is a relative ideal of $S$. Let $e_{1} \in \frac{P}{2}$ and $s \in S$; we have:

$$
e_{1}+s \in \frac{P}{2} \Leftrightarrow 2\left(e_{1}+s\right) \in P \Leftrightarrow 2 e_{1}+2 s \in P,
$$

and the last relation is true since $P \subset H, H$ is an ideal of $S \bowtie^{b} E$ and $2 e_{1}+2 s$ is even. Since $E_{1}$ is a relative ideal of $S$, it has a minimum, and therefore $\frac{P}{2}$ has a minimum too. Since $S \bowtie^{b} E$ has a conductor, it follows that $\frac{P}{2}+s \subseteq S \bowtie^{b} E$ for some $s \in S \bowtie^{b} E$.

We show now that $\frac{D-b}{2}$ is a relative ideal of $S$. Let $e_{2} \in \frac{D-b}{2}$ and $s \in S$, we have:

$$
e_{2}+s \in \frac{D-b}{2} \Leftrightarrow 2 e_{2}+2 s+b \in D
$$

and the last relation is true since $2 e_{2}+b \in D$. Arguing similarly as we did for $\frac{P}{2}$, we get that $\frac{D-b}{2}+s \subseteq S \bowtie^{b} E$, for some $s \in S \bowtie^{b} E$.

We prove now that $\frac{P}{2}$ and $\frac{D-b}{2}$ satisfy Properties 1 and 2 of Definition 3.1. If $e_{1} \in \frac{P}{2}$ and $e \in E$, then $2 e_{1}+(2 e+b)$ is an odd element of $H$, hence $2 e_{1}+2 e+b \in D$ and it follows that $e_{1}+e \in \frac{D-b}{2}$, so Property 1 is proven. If $e_{2} \in E_{2}$, then $2 e_{2}+b+2 e+b$ is an even element of $H$. Hence, $2 e_{2}+b+2 e+b \in P$ and it follows that $e_{2}+b+e \in \frac{P}{2}$, so Property 2 is proven.

Finally, we prove the unicity of $E_{1}$ and $E_{2}$. Let $E_{1}^{\prime}$ and $E_{2}^{\prime}$ be relative ideals of $S$ such that $H=E_{1}^{\prime} \bowtie^{b} E_{2}^{\prime}$. If $e_{1}^{\prime} \in E_{1}^{\prime}$, then $2 e_{1}^{\prime}$ is an even element of $H$, so $e_{1}^{\prime} \in \frac{P}{2}$. If instead $e_{1} \in \frac{P}{2}$, then $2 e_{1}$ is an even element of $H$, so $e_{1} \in E_{1}^{\prime}$. Similarly, we prove that $E_{2}^{\prime}=\frac{D-b}{2}$.

In the following, if $H$ is a relative ideal of $S \bowtie^{b} E$, then we will denote by $E_{1}(H)$ and $E_{2}(H)$, the unique relative ideals of $S$ such that $H=E_{1}(H) \bowtie^{b} E_{2}(H)$, and we will call them the even and the odd component respectively. If there is no risk of misunderstanding, we will not write $H$ within the brackets. 
If we want to compute the even component of a relative ideal of $S \bowtie^{b} E$, we have to take the even numbers of the ideal and divide them by 2 . Similarly, to compute the odd component, we have to take the odd numbers, subtract $b$ and then divide them by 2 ; by Theorem 3.4, they are indeed relative ideals of $S$. With this mindset, it is trivial to compute $M\left(S \bowtie^{b} E\right)=M \bowtie^{b} E$ and $C\left(S \bowtie^{b} E\right)=\left(C(E)+\frac{b-1}{2}\right) \bowtie^{b} C(E)$. It is fairly more interesting though to compute the components of the standard canonical ideal of $S \bowtie^{b} E$. The following result was originally proven in [9, Lemma 2.2] without using the ideal duplication.

Proposition 3.5 The following equality holds:

$$
K\left(S \bowtie^{b} E\right)=(K-\tilde{E}) \bowtie^{b}(K+e) .
$$

Proof Let $x$ be even. We have:

$x \in K\left(S \bowtie^{b} E\right) \Leftrightarrow 2 f(E)+b-x \notin 2 \cdot E+b \Leftrightarrow 2 f-x \notin 2 \cdot \tilde{E} \Leftrightarrow f-\frac{x}{2} \notin \tilde{E}$.

Since $K-\tilde{E}=\{x \in \mathbb{Z} \mid f-x \notin \tilde{E}\}$, we get $\frac{x}{2} \in K-\tilde{E}$.

Let $x$ be odd. We have:

$$
x \in K\left(S \bowtie^{b} E\right) \Leftrightarrow 2 f(E)+b-x \notin 2 \cdot S \Leftrightarrow 2 f-(x-b-2 e) \notin 2 . \quad S \Leftrightarrow \frac{x-b}{2} \in K+e,
$$

that is the thesis.

We see now how the components behave under the basic operations between ideals.

Proposition 3.6 Let I and $J$ be two relative ideals of $S \bowtie^{b} E$. The following facts are true:
a. If $I \subseteq J$, then $E_{i}(I) \subseteq E_{i}(J), i=1,2$;
b. $E_{i}(I \cup J)=E_{i}(I) \cup E_{i}(J), i=1,2$;
c. $E_{i}(I \cap J)=E_{i}(I) \cap E_{i}(J), i=1,2$.

Proof $a$ is trivial.

The proofs of $b$ and $c$ are similar, we prove $b$ as an example:

$$
E_{1}(I \cup J)=\frac{P(I) \cup P(J)}{2}=\frac{P(I)}{2} \cup \frac{P(J)}{2}=E_{1}(I) \cup E_{1}(J),
$$

where $P(I)$ and $P(J)$ are the even elements of $I$ and $J$ respectively. The proof for the odd component is similar.

Proposition 3.7 Let $x \in \mathbb{Z}$ be and let $H$ be a relative ideal of $S \bowtie^{b} E$. If $x$ is even, then

$$
E_{1}(H+x)=E_{1}(H)+\frac{x}{2} \text { and } E_{2}(H+x)=E_{2}(H)+\frac{x}{2} .
$$


If $x$ is odd, then

$$
E_{1}(H+x)=E_{2}(H)+\frac{x+b}{2} \text { and } E_{2}(H+x)=E_{1}(H)+\frac{x-b}{2} .
$$

Proof Let $x$ be an even number and let $y \in E_{1}(H+x)$; this means that there exists $h \in H$ even such that $2 y=h+x$, therefore $y=\frac{h}{2}+\frac{x}{2}$. Let $y \in E_{2}(H+x)$; this means that there exists $h \in H$ odd such that $2 y+b=h+x$, therefore $y=\frac{h-b}{2}+\frac{x}{2}$.

Let $x$ be an odd number and let $y \in E_{1}(H+x)$; this means that there exists $h \in H$ odd such that $2 y=h+x$, hence $y=\frac{h-b}{2}+\frac{x+b}{2}$. Let $y \in E_{2}(H+x)$; this means that there exists $h \in H$ even such that $2 y+b=h+x$, therefore $y=\frac{h}{2}+\frac{x-b}{2}$.

Proposition 3.8 Let $I$ and $J$ be two ideals of $S \bowtie^{b} E$, then the following equalities hold:

$$
\begin{array}{r}
E_{1}(I-J)=\left(\left(E_{1}(I)-E_{1}(J)\right) \cap\left(E_{2}(I)-E_{2}(J)\right),\right. \\
E_{2}(I-J)=\left(E_{2}(I)-E_{1}(J)\right) \cap\left(E_{1}(I)-\left(E_{2}(J)+b\right)\right) .
\end{array}
$$

Proof Let $x \in E_{1}(I-J)$; we first show that $x \in E_{1}(I)-E_{1}(J)$. For every $e_{1} \in E_{1}(J)$, we have:

$$
x+e_{1} \in E_{1}(I) \Leftrightarrow 2 x+2 e_{1} \in I .
$$

The last relation is true since $2 x \in I-J$ and $2 e_{1} \in J$. Similarly, we prove that $x \in E_{2}(I)-E_{2}(J)$.

Conversely, let $x \in\left(E_{1}(I)-E_{1}(J)\right) \cap\left(E_{2}(I)-E_{2}(J)\right)$ and $j \in J$.

(i) If $j=2 e_{1}$, with $e_{1} \in E_{1}(J)$, we have:

$$
2 x+j=2 x+2 e_{1}=2\left(x+e_{1}\right) \in I \Leftrightarrow 2 x \in I-J \Leftrightarrow x \in E_{1}(I-J) ;
$$

(ii) If $j=2 e_{2}+b$, with $e_{2} \in E_{2}(J)$, we have:

$$
2 x+j=2 x+2 e_{2}+b=2\left(x+e_{2}\right)+b \in I \Leftrightarrow 2 x \in I-J \Leftrightarrow x \in E_{1}(I-J) .
$$

The proof for the odd component is similar.

Proposition 3.9 Let I and $J$ be two relative ideals of $S \bowtie^{b} E$, the following equalities hold:

$$
\begin{aligned}
& E_{1}(I+J)=\left(E_{1}(I)+E_{1}(J)\right) \cup\left(E_{2}(I)+E_{2}(J)+b\right), \\
& E_{2}(I+J)=\left(E_{1}(I)+E_{2}(J)\right) \cup\left(E_{2}(I)+E_{1}(J)\right) .
\end{aligned}
$$


Proof Using Property 6 of Lemma 2.1, we get:

$$
\begin{gathered}
I+J=\left[2 \cdot E_{1}(I) \cup\left(2 \cdot E_{2}(I)+b\right)\right]+\left[2 \cdot E_{1}(J) \cup\left(2 \cdot E_{2}(J)+b\right)\right]= \\
=\left[2 \cdot\left(E_{1}(I)+E_{1}(J)\right)\right] \cup\left[2 \cdot\left(E_{2}(I)+E_{2}(J)+b\right)\right] \cup \\
\cup\left[2 \cdot\left(E_{1}(I)+E_{2}(J)\right)+b\right] \cup\left[2 \cdot\left(E_{2}(I)+E_{1}(J)\right)+b\right] .
\end{gathered}
$$

Since all the even numbers of the sum are within the first and the second set of the union and the odd numbers are within the last two sets of the union, we obtain the thesis.

Proposition 3.10 Let I and $J$ be two relative ideals of $S \bowtie^{b} E$, the following equality holds:

$$
I \backslash J=2 \cdot\left(E_{1}(I) \backslash E_{1}(J)\right) \cup\left(2 \cdot\left(E_{2}(I) \backslash E_{2}(J)\right)+b\right) .
$$

In particular, the cardinality of $I \backslash J$ is:

$$
\left|E_{1}(I) \backslash E_{1}(J)\right|+\left|E_{2}(I) \backslash E_{2}(J)\right|
$$

Proof Let $x \in I \backslash J$. If $x$ is even, then $\frac{x}{2} \in E_{1}(I)$, and at the same time, since $x \notin J$, it follows that $\frac{x}{2} \notin E_{1}(J)$. If $x$ is odd, the proof is similar.

For the last part of the thesis, it suffices to notice that the functions $z \mapsto 2 z$ and $z \mapsto 2 z+b$, defined in $\mathbb{Z}$, are injective.

The following result was originally proven in [7, Proposition 3.1.2] without using the ideal duplication.

Corollary 3.11 The following equality holds:

$$
g\left(S \bowtie^{b} E\right)=g(S)+g(E)+m(E)+\frac{b-1}{2}
$$

Proof Since $\mathbb{N}=C\left(S \bowtie^{b} E\right)-(2 f(E)+b+1)$, by Proposition 3.7, we get $\mathbb{N}=$ $\mathbb{N} \bowtie^{b}\left(\mathbb{N}-\frac{b-1}{2}\right)$. We have that $\mathbb{N}-\frac{b-1}{2}=B \cup \mathbb{N}$, where $B=\left\{-\frac{b-1}{2}, \ldots,-1\right\}$; moreover, $2 \cdot B+b$ is equal to the set of odd numbers between 1 and $b$. By Proposition 3.10, we have:

$$
\mathbb{N} \backslash\left(S \bowtie^{b} E\right)=2 \cdot(\mathbb{N} \backslash S) \cup\left\{1,3, \ldots, 2 h+1, \ldots, b, 2 g_{1}+b, \ldots, 2 g_{n}+b\right\},
$$

where $\mathbb{N} \backslash E=\left\{g_{1}, \ldots, g_{n}\right\}$ and $n=g(E)+m(E)$.

\section{Nearly Gorenstein duplication and applications of the ideal duplication}

In the first part of this section, we recall the definition of nearly Gorenstein semigroup and we present a characterization for $S \bowtie^{b} E$ to be nearly Gorenstein; furthermore, 
we apply this result to study the nearly Gorensteinness for numerical duplications obtained by some particular classes of ideals.

In the last part, after introducing the definition of almost symmetric semigroup, we describe the pseudo-Frobenius numbers of the numerical duplication. This allows to produce a new characterization for $S \bowtie^{b} E$ to be almost symmetric. For the rest of the paper, we exclude the trivial case $S=\mathbb{N}$.

Let $I$ be a relative ideal of $S$. We define the trace of $I$ the following ideal:

$$
\operatorname{Tr}_{S}(I)=I+(S-I)
$$

This definition has its origin in ring theory (see [11, p. 4]) and it is the anologue for the numerical semigroup case. It is straightforward to prove that the trace is independent of shifts and also that $\operatorname{Tr}_{S}(I) \subseteq S$.

The trace of $S$ is defined as the trace of its standard canonical ideal, $\operatorname{Tr}(S)=$ $\operatorname{Tr}_{S}(K)=K+(S-K) ; S-K$ is usually referred as the anti-canonical ideal of $S$.

Definition 4.1 $S$ is said to be a nearly Gorenstein semigroup if

$$
M \subseteq \operatorname{Tr}(S)=K+(S-K)
$$

This definition was originally introduced in [11, Definition 2.2]. The numerical semigroup ring associated to $S$ is nearly Gorenstein if and only if the semigroup is nearly Gorenstein. It is straightforward to prove that, if $S$ is symmetric, then it is nearly Gorenstein; in fact, the symmetric semigroups are exactly those for which the trace blows up to $S$. Hence, $S$ is nearly Gorenstein but not Gorenstein if and only if $M=K+(S-K)$; using Lemma 2.1, it is easy to prove that this condition is equivalent to $K-M=S-(S-K)$.

Theorem 4.2 The following equalities hold:

$$
\begin{aligned}
& E_{1}\left(\operatorname{Tr}\left(S \bowtie^{b} E\right)\right)=(K-E)+(E-K), \\
& E_{2}\left(\operatorname{Tr}\left(S \bowtie^{b} E\right)\right)=((K-E)+(E-(K-E))) \cup(K+(E-K)) .
\end{aligned}
$$

Proof First of all we prove that $E-(K-E) \subseteq S-(K+b)$. We have:

$$
(E-(K-E))+(K+b) \subseteq(E-K)+(K+b) \subseteq E+b \subseteq S .
$$

Using this fact, Propositions 2.7 and 3.8, we have:

$$
S \bowtie^{b} E-K\left(S \bowtie^{b} E\right)=(\tilde{E}-K) \bowtie^{b}(E-(K-\tilde{E})) .
$$

It is straightforward to prove that $(K+b)+(E-(K-E)) \subseteq(K-E)+(E-K)$. Using this fact, Property 8 of Lemma 2.1, and Proposition 3.9, we get:

$$
E_{1}\left(\operatorname{Tr}\left(S \bowtie^{b} E\right)\right)=(K-E)+(E-K) .
$$


With a similar argument, we can prove the equality for the odd component.

As direct consequence of Theorem 4.2, we get the following characterization.

Corollary 4.3 $S \bowtie^{b} E$ is nearly Gorenstein if and only if: $M \subseteq(K-E)+(E-K)$ and

$$
E=((K-E)+(E-(K-E))) \cup(K+(E-K))
$$

In particular, the nearly Gorensteinness of $S \bowtie^{b} E$ does not depend on b or on shifts of $E$.

Example 4.4 1) Let $S=\{0,3,5,6,8 \rightarrow\}, E=\{10,13, \rightarrow\}$. We have:

$$
(K-E)+(E-K)=\{5,6,8, \rightarrow\} \subset M,
$$

but

$$
((K-E)+(E-(K-E))) \cup(K+(E-K))=\{10,13 \rightarrow\}=E .
$$

Hence, the first condition of Corollary 4.3 is independent of the second one.

2) Let $S=\{0,4,7,8,10, \rightarrow\}, E=\{4,8,11,12,14, \rightarrow\}$. We have:

$$
(K-E)+(E-K)=\{4,7,8,10, \rightarrow\}=M
$$

but

$$
((K-E)+(E-(K-E))) \cup(K+(E-K))=\{8,11,12,14, \rightarrow\} \subset E,
$$

Hence, the second condition of Corollary 4.3 is independent of the first one.

Proposition 4.5 If $M \subseteq(K-E)+(E-K)$, then:

1. $M-M \supseteq(E-K)-(E-K)$;

2. $K-M \supseteq E-(E-K)$.

In particular, $E-E \subseteq M-M$.

Proof Both statements 1 and 2 are consequence of Property 4 of Lemma 2.1, Property 1 of Corollary 2.3 and Proposition 2.7. The last part is true since:

$$
E-E \subseteq E-(K+(E-K))=(E-K)-(E-K) \subseteq M-M .
$$

It is worth noticing that if $M=(K-E)+(E-K)$, then both 1 and 2 of the previous proposition become equalities.

We recall that $s_{1}$ denotes the multiplicity of $S$, i.e. the minimum of $M$.

Lemma 4.6 If $S \bowtie^{b} E$ is nearly Gorenstein and not symmetric, then $s_{1}=m(\tilde{E}-K)$, i.e. $s_{1}+K \subset \tilde{E} \subset K$. 
Proof Clearly $s_{1}=m(K-\tilde{E})+m(\tilde{E}-K)$. Using Lemma 2.6, we get $m(K-\tilde{E})=$ $f(K)-f(\tilde{E})=f-f=0$.

Note that, if $\tilde{E} \neq K$, then $s_{1} \in \tilde{E}-K$ if and only if $s_{1}=m(\tilde{E}-K)$.

We now apply the above results to some particular cases.

Corollary 4.7 If $S$ is symmetric, then:

$$
\operatorname{Tr}\left(S \bowtie^{b} E\right)=[(S-E)+E] \bowtie^{b} E .
$$

In particular, if $S$ is symmetric, then $S \bowtie^{b} E$ is nearly Gorenstein if and only if $\operatorname{Tr}_{S}(E) \supseteq$ M.

Proof By Theorem 4.2, we see that $E-K \subseteq E_{2}\left(\operatorname{Tr}\left(S \bowtie^{b} E\right)\right) \subseteq E$. Since $S$ is symmetric, then $E-K=E-S=E$, that is the thesis.

Corollary 4.8 If $K-\tilde{E}$ is a numerical semigroup, then:

$$
\operatorname{Tr}\left(S \bowtie^{b} E\right)=\operatorname{Tr}_{S}(E-E) \bowtie^{b} E .
$$

Proof Using Theorem 4.2, by Corollary 2.8 and Corollary 2.9, if follows easily that

$$
E_{1}\left(\operatorname{Tr}\left(S \bowtie^{b} E\right)\right)=(E-E)+(S-(E-E)) .
$$

Using Theorem 4.2 to compute the odd component of $\operatorname{Tr}\left(S \bowtie^{b} E\right)$, it suffices to show that Corollary 2.9 implies that:

$$
(K-\tilde{E})+(E-(K-\tilde{E}))=(E-E)+(E-(E-E))=E .
$$

Proposition 4.9 The following equality holds:

$$
\operatorname{Tr}\left(S \bowtie^{b} M\right)=(\operatorname{Tr}(S) \cap M) \bowtie^{b}(\operatorname{Tr}(S) \cap M) .
$$

In particular, $S \bowtie^{b} M$ is nearly Gorenstein if and only if $S$ is nearly Gorenstein.

Proof If $S$ is symmetric, then, by Corollary 4.7, we get:

$$
\operatorname{Tr}\left(S \bowtie^{b} M\right)=((S-M)+M) \bowtie^{b} M=M \bowtie^{b} M=(\operatorname{Tr}(S) \cap M) \bowtie^{b}(\operatorname{Tr}(S) \cap M) .
$$

Assume $S$ to be not symmetric, hence $S-K=M-K$. In fact, let $x \in S-K$ and $y \in K$. If $x+y=0$, then $x<0$ which is a contradiction because $S-K \subset S$. By Theorem 4.2, using Property 6 of Lemma 2.1 and Proposition 2.5, we get:

$$
\begin{aligned}
E_{1}\left(\operatorname{Tr}\left(S \bowtie^{b} M\right)\right)= & (K-M)+(M-K)=(K \cup\{f\})+(M-K)= \\
& (K+(M-K)) \cup((M-K)+f) .
\end{aligned}
$$


Since $(M-K)+f \subseteq C \subseteq K+(M-K)$ and $M-K=S-K$, we get:

$$
E_{1}\left(\operatorname{Tr}\left(S \bowtie^{b} M\right)\right)=K+(M-K)=K+(S-K)=\operatorname{Tr}(S)=\operatorname{Tr}(S) \cap M .
$$

With a similar argument done at the beginning of the proof, we can prove that $M-(K-M)=S-(K-M)$ (this equality holds also in the case that $S$ is symmetric). Using this fact, Corollary 2.8 and using Theorem 4.2 to compute the odd component of the trace of $S \bowtie^{b} M$, we get:

$$
\begin{aligned}
& E_{2}\left(\operatorname{Tr}\left(S \bowtie^{b} M\right)\right)=((K-M)+(M-K)) \cup(K+(M-K))= \\
& (K-M)+(M-K)=E_{1}\left(\operatorname{Tr}\left(S \bowtie^{b} M\right)\right) .
\end{aligned}
$$

The following definition is a specialization of the notion of integrally closed ideal for numerical semigroup rings.

Definition 4.10 An ideal $I$ is said to be integrally closed if there exists $a \in S$ such that

$$
I=\{s \in M \mid s \geq a\} .
$$

The maximal ideal $M$ is clearly integrally closed.

Theorem 4.11 If $E$ is integrally closed and not maximal, then $S \bowtie^{b} E$ is nearly Gorenstein if and only if $s_{1}=f+1$.

Proof Assume $s_{1} \neq f+1$. If $E=\{s \in M \mid s \geq a\}$, we have two possibilities:

1. If $a \leq f+1$, then $\tilde{E}=E$. Since $s_{1} \notin E$, by Lemma $4.6, S \bowtie^{b} E$ is not nearly Gorenstein.

2. If $a>f+1$, then $\tilde{E}=E-e$. We show now that $s_{1}+e \notin E$. If $a=f+1+x$, with $x>0$, then $e=f+x-f=x$. If we suppose that $s_{1}+x \in E$, then $s_{1}+x \geq f+1+x$, and hence $s_{1} \geq f+1$; it follows that $s_{1}=f+1$, a contradiction. Since $s_{1}+e \notin E$, we get $s_{1} \notin E-e$, and once again, by Lemma 4.6, $S \bowtie^{b} E$ is not nearly Gorenstein.

Conversely, if $s_{1}=f+1$, we can write $S=\left\{0, s_{1}, \rightarrow\right\}$ and, since $E$ is integrally closed, $E$ is a shift of the maximal ideal. It is straightforward to prove that $S$ is nearly Gorenstein, so by Proposition 4.9 and using the fact that the nearly Gorensteinness is independent of shifts of $E$, we have that $S \bowtie^{b} E$ is nearly Gorenstein.

By the definition of pseudo-Frobenius numbers, it is obvious that

$$
\operatorname{PF}(S)=(S-M) \backslash S \text {. }
$$

If $S \neq \mathbb{N}$, then $S-M=M-M$, hence the pseudo-Frobenius numbers are exactly the numbers in $(M-M) \backslash S$. 
Theorem 4.12 The following equality holds:

$$
\mathrm{PF}\left(S \bowtie^{b} E\right)=2 \cdot[((M-M) \cap(E-E)) \backslash S] \cup[2 \cdot((E-M) \backslash E)+b] .
$$

In particular, $t\left(S \bowtie^{b} E\right)=|(M-M) \cap(E-E)|+|(E-M) \backslash E|$.

Proof Since $E-M \subseteq(S-E)-b$, by Proposition 3.8, it follows

$$
\left(S \bowtie^{b} E\right)-\left(M \bowtie^{b} E\right)=((M-M) \cap(E-E)) \bowtie^{b}(E-M) .
$$

By Proposition 3.10, it follows

$$
\begin{aligned}
& \left(\left(S \bowtie^{b} E\right)-\left(M \bowtie^{b} E\right)\right) \backslash\left(S \bowtie^{b} E\right) \\
& \quad=2 \cdot[((M-M) \cap(E-E)) \backslash S] \cup[2 \cdot((E-M) \backslash E)+b] .
\end{aligned}
$$

The formula for the type of $S \bowtie^{b} E$ was originally proven in [7, Proposition 3.5] without using the ideal duplication.

$S$ is said to be almost symmetric if and only if $\mathrm{PF}(S)=(K-M) \backslash S$ (i.e. $K-M$ is a numerical semigroup). This definition was originally introduced in [4] with the aim to generalize the notion of symmetric semigroups and consequently, with the introduction of almost Gorenstein rings, the notion of Gorenstein rings. In fact, every symmetric semigroup is almost symmetric. Moreover, in [11, Proposition 6.1] the authors show that any one-dimensional almost Gorenstein ring is nearly Gorenstein, and therefore any almost symmetric semigroup is nearly Gorenstein.

Corollary 4.13 $S \bowtie^{b} E$ is almost symmetric if and only if: $K-\tilde{E}=(M-M) \cap(E-E)$ and $K-M=\tilde{E}-M$. In particular, the almost symmetry of $S$ does not depend on $b$ or shifts of $S$.

\section{Proof Since}

$\left(K\left(S \bowtie^{b} E\right)-M\left(S \bowtie^{b} E\right)\right) \backslash\left(S \bowtie^{b} E\right)=2 \cdot((K-\tilde{E}) \backslash S) \cup(2 \cdot((K+e)-M)+b)$,

by Theorem 4.12 and the definition of almost symmetric semigroup, the statement follows.

The following result was originally proven in [7, Theorem 4.3]. We present an alternative proof using Corollary 4.13 .

Theorem 4.14 $S \bowtie^{b} E$ is almost symmetric if and only if

$$
K-(M-M) \subseteq \tilde{E} \subseteq K
$$

and $K-\tilde{E}$ is a numerical semigroup. In particular, the almost symmetry of $S \bowtie^{b} E$ does not depend on $b$ or shifts of $E$. 
Proof If $S \bowtie^{b} E$ is almost symmetric, then, by the first equality of Corollary 4.13, $K-\tilde{E}$ is a numerical semigroup; furthermore, by Corollary 2.9, it is also true that $K-\tilde{E}=E-E$. Hence, $K-\tilde{E} \subseteq M-M$, and therefore $K-(M-M) \subseteq \tilde{E} \subseteq K$.

Conversely, we want to prove that $S \bowtie^{b} E$ is almost symmetric using Corollary 4.13. Since $K-\tilde{E}$ is a numerical semigroup and $K-(M-M) \subseteq \tilde{E}$, by Corollary 2.9, it follows that $E-E \subseteq M-M$, therefore $K-\tilde{E}=(M-M) \cap(E-E)$. Since $\tilde{E}-M \subseteq K-M$ is easily proven, we only need to show the converse inclusion. By Property 5 of Lemma 2.1, we get:

$$
K-M \subseteq K-((M-M)+M) \subseteq(K-(M-M))-M \subseteq \tilde{E}-M .
$$

Using Theorem 4.14, it is trivial to prove the following:

Corollary 4.15 $S \bowtie^{b} M$ is almost symmetric if and only if $S$ is almost symmetric.

In the case of almost symmetric numerical duplication, we are able to describe the pseudo-Frobenius numbers of $S \bowtie^{b} E$ with a greater degree of precision.

Theorem 4.16 Suppose $S \bowtie^{b} E$ to be almost symmetric. Then, the following is true:

$$
\operatorname{PF}\left(S \bowtie^{b} E\right)=2 \cdot[(E-E) \backslash S] \cup[2 \cdot((K+e) \backslash E)+b] \cup\{2 f(E)+b\} .
$$

Moreover, if $\tilde{E} \neq K$, then $S \bowtie^{b} E$ always has at least one even pseudo-Frobenius number, and the even pseudo-Frobenius numbers do not depend on $b$ or shifts of $E$.

Proof Using Property 7 of Lemma 2.1, by Corollary 4.13 and Proposition 2.5, we get:

$$
\begin{aligned}
& (E-M) \backslash E=((K-M) \backslash \tilde{E})+e=((K+e) \cup\{f(E)\}) \backslash E \\
& \quad=((K+e) \backslash E) \cup\{f(E)\} .
\end{aligned}
$$

Using Theorem 4.12 and Proposition 4.5, we get the thesis.

For the last part, the only non-trivial thing to prove is that, if $\tilde{E} \neq K$, then $E-E \neq$ $S$. In fact, if $E-E=S$, by Theorem 4.14 and Corollary 2.9, we would get that $K=K-(E-E)=K-(K-\tilde{E})=\tilde{E}$.

The following result was originally proven in [7, Proposition 4.8]; we present an alternative proof using Theorem 4.16.

Corollary 4.17 Suppose $S \bowtie^{b} E$ to be almost symmetric. Then we have:

$$
t\left(S \ltimes^{b} E\right)=2|(E-E) \backslash S|+1=2|K \backslash \tilde{E}|+1 .
$$

In particular, the type of $S \bowtie^{b} E$ is always odd and $1 \leq t(S) \leq 2 t(S)+1$.

Proof By Property 7 of Corollary 2.1, we get that $|2 \cdot((K+e) \backslash E)+b|=|K \backslash \tilde{E}|$. Moreover, by Property 5 of Corollary 2.3 and Corollary 2.9, it follows that $\mid(E-E) \backslash$ $S|=| K \backslash \tilde{E} \mid$. Using Theorem 4.16, we get

$$
t\left(S \bowtie^{b} E\right)=|(E-E) \backslash S|+|K \backslash \tilde{E}|+1=2|K \backslash \tilde{E}|+1 .
$$


The last part follows from the fact that, by Proposition 4.5, if $S \bowtie^{b} E$ is almost symmetric (or also if it is nearly Gorenstein), then $E-E \subseteq M-M$.

In [7,p.159], the authors prove that it is possible to obtain any odd integer $x=2 m+1$ in the range described in the previous corollary.

Example 4.18 The previous corollary states that, in almost symmetric numerical duplications, the type is always odd; hence, it is natural to ask if this fact is true in the nearly Gorenstein case. In general this is not the case; in fact, take for example $S=\{0,4,7,8,10, \rightarrow\}$ and $E=\{3,6,7,10,11,13, \rightarrow\}$. Then $S \bowtie^{b} E$ is nearly Gorenstein, but $t\left(S \bowtie^{b} E\right)=2$. Furthermore, since $E-E=S$, the pseudo-Frobenius numbers are all odd.

Proposition 4.19 Let E be a principal ideal (i.e. $E=s+S$, with $s \in S$ ). The following facts are equivalent:

1. S is symmetric;

2. $S \bowtie^{b} E$ is symmetric;

3. $S \bowtie^{b} E$ is almost symmetric;

4. $S \bowtie^{b} E$ is nearly Gorenstein.

Proof The implications $1 \Rightarrow 2,2 \Rightarrow 3$ and $3 \Rightarrow 4$ are trivial; we only need to prove that $4 \Rightarrow 1$.

If $S \bowtie^{b} E$ is nearly Gorenstein, using Theorem 4.2 to compute the trace, we get:

$$
\operatorname{Tr}\left(S \bowtie^{b} E\right)=\operatorname{Tr}(S) \bowtie^{b}(s+\operatorname{Tr}(S)) \supseteq M \bowtie^{b}(s+S) .
$$

Therefore, $\operatorname{Tr}(S)=S$, and hence $S$ is symmetric.

Example 4.20 Since we proved that, if $E$ is principal, then $S \bowtie^{b} E$ is almost symmetric if and only if it is nearly Gorenstein, it is natural to ask if for any 2-generated ideal $E$, the equivalence still holds. The answer, in general, is no.

Let $S=\{0,6,7,9,12,13,14,15,16,18, \rightarrow\}$ and $E=\{6,12,13,15,18, \rightarrow\}=$ $(6,23)+S$. Since $S$ is symmetric, using Corollary 4.7 to compute the trace, we get that $S \bowtie^{b} E$ is nearly Gorenstein. On the other hand, since $\tilde{E}=E \subset M=K-(M-M)$, by Theorem 4.14, $S$ is not almost symmetric.

Example 4.21 Let $S=\{0,4,7,8,10, \rightarrow\}$. We have $K=\{0,3,4,6,7,8,10, \rightarrow\}$ and $K-(M-M)=M$. All the ideals between $M$ and $K$ are listed below:

- $\tilde{E}_{0}=M$,

- $\tilde{E}_{1}=\{0,4,7,8,10, \rightarrow\}=S$,

- $\tilde{E}_{2}=\{3,4,7,8,10, \rightarrow\}$,

- $\tilde{E}_{3}=\{4,6,7,8,10, \rightarrow\}$,

- $\tilde{E}_{4}=\{0,3,4,7,8,10, \rightarrow\}$,

- $\tilde{E}_{5}=\{0,4,6,7,8,10, \rightarrow\}$,

- $\tilde{E}_{6}=\{3,4,6,7,8,10, \rightarrow\}$,

- $\tilde{E}_{7}=K$. 
Among these ideals, only $\tilde{E}_{1}$ and $\tilde{E}_{2}$ do not give rise to nearly Gorenstein numerical duplications. While $\tilde{E}_{4}$ is the only one that gives rise to nearly Gorenstein numerical duplication but not almost symmetric (because $K-\tilde{E}_{4}$ is not a numerical semigroup).

Unfortunately, it is much harder to determinate the family of class of shifts for the nearly Gorensteinness of the numerical duplication. Thanks to Lemma 4.6, we can say that $\tilde{E}$ must be between $s_{1}+K \subset \tilde{E} \subseteq K$ (do note that $s_{1}+K \subset K-(M-M)$ ). In the example, since $s_{1}+K=M \backslash\{13\}$, we can say that we already found all the possible classes of shifts for which $S \bowtie^{b} E$ is nearly Gorenstein.

Acknowledgements I would like to thank professor Marco D'Anna for his help during the writing of the paper and for all the useful tips and suggestions during the first years of my $\mathrm{PhD}$ course. I would like to thank the anonimous referee for his/her careful reading of the manuscript and for his/her suggestions.

Funding Open access funding provided by Università degli Studi di Catania within the CRUI-CARE Agreement.

Open Access This article is licensed under a Creative Commons Attribution 4.0 International License, which permits use, sharing, adaptation, distribution and reproduction in any medium or format, as long as you give appropriate credit to the original author(s) and the source, provide a link to the Creative Commons licence, and indicate if changes were made. The images or other third party material in this article are included in the article's Creative Commons licence, unless indicated otherwise in a credit line to the material. If material is not included in the article's Creative Commons licence and your intended use is not permitted by statutory regulation or exceeds the permitted use, you will need to obtain permission directly from the copyright holder. To view a copy of this licence, visit http://creativecommons.org/licenses/by/4.0/.

\section{References}

1. Assi, A., D’Anna, M., García-Sánchez, P.A.: Numerical semigroups and applications. RSME Springer Ser. (2020). https://doi.org/10.1007/978-3-030-54943-5

2. Barucci, V., D’Anna, M., Strazzanti, F.: A family of quotients of the rees algebra. Commun. Algebra 43(1), 130-142 (2015). https://doi.org/10.1080/00927872.2014.897549

3. Barucci, V., D’Anna, M., Strazzanti, F.: Families of Gorenstein and almost Gorenstein rings. Ark. Mat. 54, 321-338 (2016). https://doi.org/10.1007/s11512-016-0235-5

4. Barucci, V., Fröberg, R.: One-dimensional almost Gorenstein rings. J. Algebra 188, 418-442 (1997). https://doi.org/10.1006/jabr.1996.6837

5. D’Anna, M.: A construction of Gorenstein rings. J. Algebra 306, 507-519 (2006). https://doi.org/10. 1016/j.jalgebra.2005.12.023

6. D'Anna, M., Fontana, M.: An amalgamated duplication of a ring along an ideal: the basic properties. J. Algebra Appl. 6, 443-459 (2007). https://doi.org/10.1142/S0219498807002326

7. D'Anna, M., Strazzanti, F.: The numerical duplication of a numerical semigroup. Semigroup Forum. 87, 149-160 (2013). https://doi.org/10.1007/s00233-012-9451-x

8. Fossum, R., Griffith, P., Reiten, I.: Trivial Extensions of Abelian Categories. Homological Algebra of Trivial Extensions of Abelian Categories with Applications to Ring Theory. Lecture Notes in Mathematics, vol. 456. Springer, Berlin (1975)

9. Frigenti, F.: Canonical reduction for quadratic quotients of the Rees algebra. Le Matematiche 74(2), 397-409 (2019). https://doi.org/10.4418/2019.74.2.11

10. Fröberg, R., Gottlieb, C., Häggkvist R.: Semigroups, semigroup rings and analytically irreducible rings. Report Dept. Math. Univ. Stockholm, no. 1 (1986)

11. Herzog, J., Hibi, T., Stamate, D.I.: The trace of the canonical module. Isr. J. Math. 233, 133-165 (2019). https://doi.org/10.1007/s11856-019-1898-y 
12. Jäger', J.: Längenberechnung und kanonische Ideale in eindimensionalen Ringen. Arch. Math. 29, 504-512 (1977). https://doi.org/10.1007/BF01220445

13. Nagata, M.: Local Rings. Interscience Publishers, New York (1962)

Publisher's Note Springer Nature remains neutral with regard to jurisdictional claims in published maps and institutional affiliations. 